\title{
Better Scalable Routing Protocol FOR HYBRID WIRELESS MESH NETWORK
}

\author{
Debraj Modak ${ }^{1}$ and Dipankar Saha ${ }^{2}$ \\ ${ }^{1}$ Department of Electronics and Communication Engineering, \\ Abacus Institute of Engineering and Management, Mogra, Hooghly. \\ ddebraj.hetc@gmail.com \\ ${ }^{2}$ Department of Electronics and Communication Engineering, \\ Global Institute of Management and Technology, Krishnanagar, Nadia. \\ dipankar.hetc@gmail.com
}

\begin{abstract}
There are many routing approaches have been borrowed from mobile ad hoc network to achieve routing solutions in wireless mesh network. WMN was developed for reliable data communication and load balancing. AODV provides loop-free routes even while repairing broken links. This paper have been proposed an improved hierarchical AODV routing protocol (IH-AODV), which exhibits better scalability and performance in the network. This IH-AODV protocol has been proposed for improvement in the scaling potential of AODV. MAODV allows each node in the network to send out multicast data packets, used for multicast traffic. The wireless mesh network architecture provides reduction in installation cost, large scale deployment, reliability and self management. It is mainly focused on implementing military or specialized civilian applications. Two protocols MAODV and IH-AODV were simulated using NS2 package. Simulation results will demonstrate that, IH-AODV scales well for large network and other metrics are also better than or comparable to MAODV in hybrid WMNs.
\end{abstract}

\section{KEYWORDS}

Wireless Mesh Networks, Routing Protocols, Hierarchical, IH-AODV, MAODV

\section{INTRODUCTION}

Wireless cellular systems have been in use since 1980s. We have seen their evolutions to first, second and third generation's wireless systems. These systems work with the support of a centralized supporting structure such as an access point. The wireless users can be connected with the wireless system by the help of these access points, when they roam from one place to the other. Easy and fast deployment of wireless networks will be expected by the future generation wireless systems. A wireless mesh network [1] is a growing new technology that will allow users to access services and information electronically, irrespective of their geographic position. Wireless networks can be classified in two types: - infrastructure network and infrastructure less (ad hoc) networks. Infrastructure network consists of a network with fixed and wired gateways. A mobile host interacts with a bridge in the network (called base station) within its communication radius. The mobile unit can move geographically while it is communicating. When it goes out of range of one base station, it connects with new base station and starts communicating through it. This is called handoff. In this approach the base stations are fixed. Wireless mesh networks are becoming increasingly popular as they provide flexibility, mobility support, and are easy to

Rupak Bhattacharyya et al. (Eds) : ACER 2013, 
deploy. WMNs are usually considered to have low (or no) node mobility, where individual nodes in the WMN are usually not considered to be energy constrained.

Proactive protocols propagate topology information periodically and find routes continuously, while reactive protocols as Dynamic Source Routing protocol (DSR) [6] and Ad-hoc On-demand Distance Vector routing protocol (AODV) [4] find routes on demand. Generally, reactive protocols outperform proactive protocols in terms of packet delivery ratio, routing overhead and energy efficiency. However, in flat routing protocols, a route is maintained at the grain of the whole path, i.e., from the source to the destination. This approach works well for small networks where routes are typically short. When the network grows, routes become longer and break more often. If routes are still maintained at the grain of the whole path, the routing overhead will be high because new routes are discovered and discarded frequently. The problems mentioned above are inherent in flat routing protocols. Hierarchical routing protocols are developed to solve the problems in flat routing protocols. In these protocols, the network is divided into groups and a subset of nodes is assigned special functionalities, typically, coordinating roles. A number of routing protocols have been proposed for WMNs. We choose hybrid network because it works in both i.e. wired network as well as wireless network. A number of routing protocols have been proposed for WMNs.

Cluster head-Gateway Switch Routing (CGSR) [8] is a typical hierarchical routing protocol. CGSR divides the network into clusters that are circular regions with the radius of a predefined hop count. Way Point Routing (WPR) [5] is an improved hierarchical routing protocol, which maintains a hierarchy only for active routes, and a number of intermediate nodes on a route are selected as way points and the route is divided into segments by the way points.

Characteristics of WMN such as rapid deployment and self configuration make WMN suitable for transient on-demand network deployment scenarios. The form of mesh networks that are of most commercial interest are often called hybrid mesh networks [2]. We combine CGSR and WPR in AODV as an improved hierarchical routing protocol. We term this instantiation as an improved hierarchical AODV routing protocol (IH-AODV).

Compared to MANETs, nodes in WMNs have relatively fixed positions and communicate to the Internet through one or more gateways. Though traditional MANETs routing algorithms can be used in WMNs, their performance is typically less than ideal.

\section{The IH-AODV Routing Protocol IN WMNS}

\subsection{Motivation:}

WMNs were developed for reliable data communication and load balancing. As the mobility of many nodes in WMNs is low, if we could decrease the active nodes during the route discovery phase [3] by dividing the nodes into cluster, the delay before sending a packet may be shorten and the scalability problem also be solved because new nodes are more easy to join due to the cluster mechanism. The design goal of IH-AODV routing protocol is to improve the scaling potential of AODV when keep the merits of it. The main features of IH-AODV include:

\subsubsection{Fresh Route Detection:}

This is a novel technique for IH-AODV. It designs for quick routing discovery for the nodes which join the cluster, and can increase speed and efficiency of the route discovery. During the route maintain, it is also useful. The details will be presented in the following sections. 


\subsubsection{Coexist with AODV:}

AODV is a widely accepted protocol. There are several implements version of AODV reported. One of the important principles in designing IH-AODV is the coexistence with AODV, it means nodes that implement AODV [4] protocol can work collaboratively with nodes that implement our protocol in the same network. To achieve this, we keep all AODV route control packets, and add some new control packets as need. In fact, The IH-AODV Routing Protocol in WMNs. we marked the nodes that is static in order to minimal the discovery delay.

\subsubsection{Local Route Repair:}

However, it is suitable for situations where link failures occur near the destination. As a network growing, routes between sources and destinations become longer; this way could not work well anymore. IH-AODV let the node upstream of the break link perform a local repair instead of issuing the RERR. If the local repair is successful, a RREP will be returned either by the destination or by a node with a valid route to the destination. In the event that a RREP is not returned, the local repair is not successful and a RERR message is sent to the source node.

\subsection{IH-AODV Routing scheme:}

\subsubsection{Route Discovery:}

Our proposed mechanism is for hybrid wireless Mesh networks. Some assumptions are as following: first of all, as there are many static nodes in the networks, we define them as way point nodes (WP); other nodes are termed cluster member nodes (CM). Second, at the beginning of the route discovery, we suppose there is an initialized cluster. The WP nodes are function nodes in a cluster; they have the same characteristic as in traditional AODV but maintain a cluster member list table (CMLT). In every cluster, there are two WP nodes named start-WP and end-WP. Two neighboring clusters share a common WP node, which acts as the end-WP node of the upstream cluster and the start-WP node of the downstream cluster. The value of fresh level is used to determine the freshest path used in cluster, which just like the sequence number used in entire network in AODV to determine the freshest route to a destination. We define the mechanism above as fresh route detection. The cluster member nodes are normal nodes worked as following: when a normal node requires a route for data forwarding, it sends small 'Hello' packets to its hop count neighbor nodes, WP nodes receives the message and then check the node is in the CMLT or not, if not, feedback a reactive message (RM) to invite the node join into the cluster, while the node receive the start-WP node and the end-WP node's RM respectively, it sends join request (JR) and join into the cluster. Then the WP node works as in AODV to initiate the route discovery phase. Fig. 1 gives an example of how source node $\mathrm{S}$ finds the route to destination node d. We select nodes S, A, B and C as WP nodes and the source node S is just included. Nodes from $\mathrm{d}$ to $\mathrm{l}$ are $\mathrm{CM}$ nodes and the destination node $\mathrm{d}$ is included. A cluster can be simply identified by its start and end WP nodes. In fig. 1, there are three clusters: S-e/f-A, A-g/h/i-B and $\mathrm{B}-\mathrm{j} / \mathrm{k} / \mathrm{l} / \mathrm{d}-\mathrm{C}$. To distinguish WP nodes from CM nodes, we use uppercase letters to label WP nodes and lowercase letters to $\mathrm{CM}$ nodes. Following the route discovery schemes above, a route can be found for example: S-A-h-B-d. 


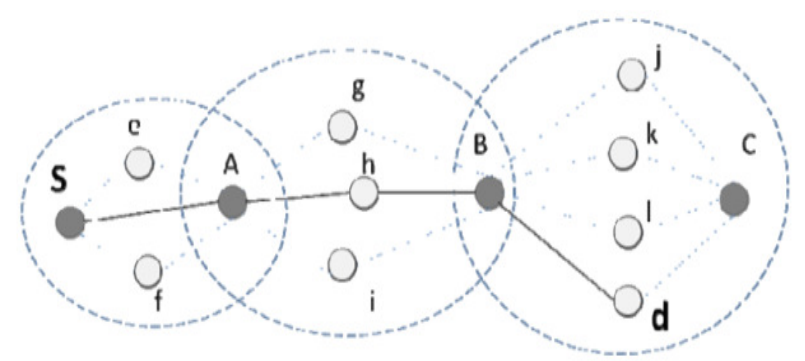

Figure 1. Route Discovery

\subsubsection{Route Maintenance}

Due to the dynamic characteristic of CM nodes, links on a route may fail. Route maintenance is the mechanism to handle route breaks. IH-AODV extends many features from AODV for route maintenance, for example, use RERR message to inform the nodes affected by the link breakage. In addition to, IH-AODV add some cluster related maintenance operations, include fresh route detect and local route repair.

Fresh route detection is not only used for route discovery but also for route maintenance. When a link failure occurs, the hop count WP node checks its CMLT firstly, and then finds the freshest path except the break one.

If this way cannot work because of the update of the CMLT, local repair will be the second level of route repair. The update of the CMLT means that the old cluster may be changed, local route repair is tried and it works in the range of one cluster [9]. The local route repair tries to establish a new path between the start and the end WP nodes of a broken cluster. Fig. 2 shows an example in which the original path for cluster $\mathrm{A}-\mathrm{g} / \mathrm{h} / \mathrm{i}-\mathrm{B}$ is $\mathrm{A}-\mathrm{h}-\mathrm{B}$ and the link between $\mathrm{h}$ and $\mathrm{B}$ breaks. As fresh route detection succeeds and the new path for cluster A-g/h/i-B is A-g-B. So a new route is established as S-A-g-B-d.

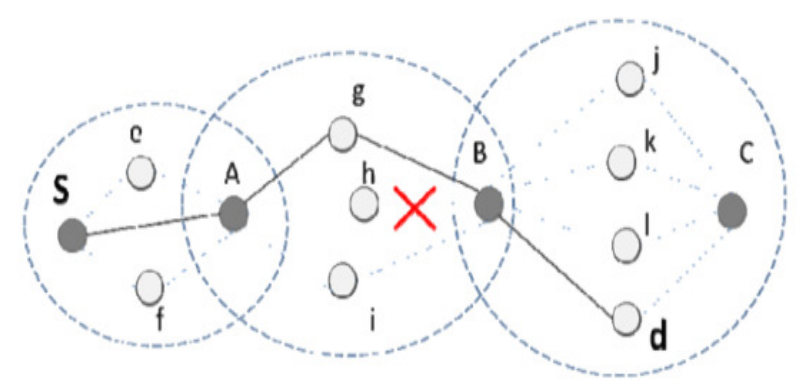

Figure 2. Route Repair

\section{MAODV Routing Protocol}

MAODV is the multicast extension of AODV. Both AODV and MAODV are routing protocols for ad-hoc networks, with AODV for unicast traffic and MAODV for multicast traffic. MAODV allows each node in the network to send out multicast data packets, and the multicast data packets are broadcast when propagating along the multicast group tree. In MAODV[7][10], each multicast group is organized by using a tree structure, composed of the group members and several routers, which are not group member but must exist in the tree to connect the group 
members. The group members and the routers are all tree members and belong to the group tree. Associated with each multicast tree, the group member that first constructs the tree is the group leader for that tree, responsible for maintaining the group tree by periodically broadcasting Group-Hello (GRPH) messages in the whole network. The group leader also maintains the group sequence number, which is propagated in the network through the GRPH. Each node in the network may maintain three tables. Unicast route table record the next hop for routes to other destinations for unicast traffic. Usually, the destination is one of the other nodes in the network. Multicast route table listing the next hops for the tree structure of each multicast group. Each entry represents one group tree structure. Every node that belongs to that group tree should maintain such entries, with its own identity as group leader, group member, or router (nonmulticast member that is in the tree to provide connectivity).Every next hop is associated with direction either downstream or upstream. If the next hop is one-hop nearer to the group leader, the direction is upstream; otherwise, the direction is downstream. The group leader has no upstream, while other nodes in the tree should have one and only one upstream. The third table is the Group leader table. It records the currently-known multicast group address with its group leader address and the next hop towards that group leader when a node receives a periodic GRPH message.

\subsection{Route Discovery and Maintenance}

In MAODV, every node in the network can send out multicast traffic, we must consider how these data packets reach each multicast group member if the data source node is not a tree member. We choose a two-step approach: first step, there is a route from that data source node to a tree member; then after the tree member receives the multicast data packets, it propagates the data through the whole tree, reaching every group member. We borrow the mechanism used for route discovery and maintenance for reaching a specific node in AODV to accomplish the first step. The data source node initiates a RREQ to ask for a route to that multicast address. Usually, this RREQ is the same as the RREQ used in AODV, without any flags and broadcasted in the network. But with the Group leader table, the source node may already know a route to reach the group leader. By using the information recorded in the Group leader table, the RREQ can be sent unicastly towards the group leader if this is the first time the node sends RREQ. During RREQ propagation, the reverse route towards the source node is constructed. Any node not in the multicast tree but with fresh enough route to that multicast address, or any tree member with known group leader can respond to this RREQ with a RREP. While the RREP is sent back to the source node along the reverse route, every intermediate node and the source node updates the route to that tree member with the destination address set to the multicast group address, thus the forwarding route is established in their Unicast route tables. For this first step, the end node is a tree member. The second step is accomplished under multicast tree construction.

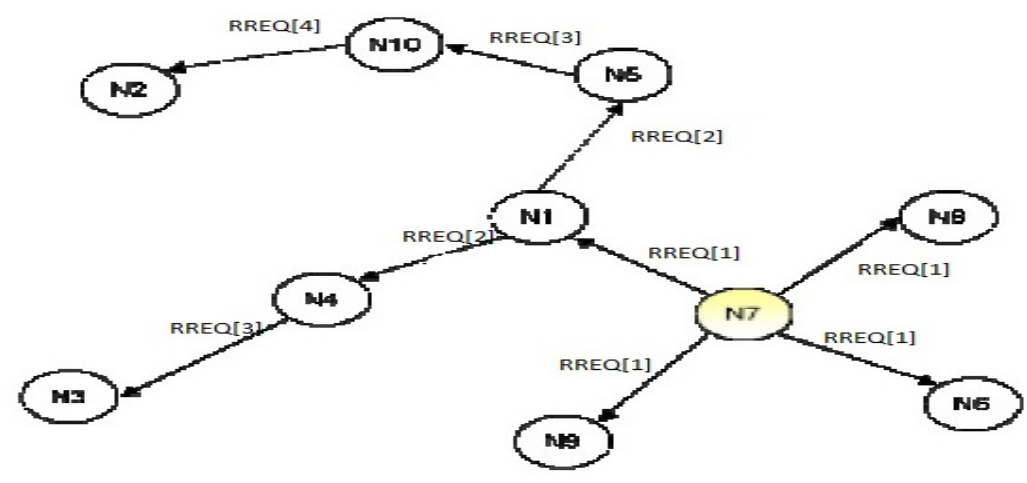

Figure 3: Initial RREQs 


\subsection{Multicast Tree Construction}

The same RREQ and RREP messages used in AODV are adapted to be used for tree construction in MAODV. A node initiates a RREQ with a join flag (RREQ-J) when it is not a tree member and wants to join that multicast group. Before sending out RREQ-J, the node creates an entry in its Multicast route Table, and identifies itself as a group member, but with an unknown group leader address, and without any upstream and downstream next hop. If a node in the tree but not a group member wants to become a group member, it simply changes its identity recorded in its Multicast route table, from a router to a group member. Usually, RREQ-J is broadcast in the network, but if the node can get information about the group leader and how to reach that group leader through checking its own Group Leader Table, and this is the first time that it sends out RREQ-J, RREQ-J can be sent unicastly towards the group leader. During RREQ-J propagation, the reverse route back to the request source node is established in the unicast route table. Multicast Route Activation (MACT), is used for grafting a branch to the tree. When the request source node sends out RREQ-J and waits for a specific time RREP_WAIT_TIME, it checks if it already received any RREP-J and cached the corresponding information. If it has the information, it sends out a MACT with a join flag (MACT-J) towards the cached upstream node and adds the new next hop in its Multicast route table. Every node that receives MACT-J should add a new next hop indicating the downstream from where it receives the MACT-J in its Multicast route table. Then if it is a tree member, the branch is finally grafted; otherwise, it will check its own cache to find the potential upstream next hop towards the tree, add this next hop in its Multicast route table, and send out a MACT-J towards that node as well.

\subsection{Multicast Tree Maintenance}

Multicast tree maintenance Periodic Group-Hello Propagation, Neighbor Connectivity Maintenance, Group Leader Selection and Tree Marge.

\subsubsection{Periodic Group-Hello Propagation}

The group leader must periodically broadcast a Group-Hello message (GRPH) throughout the whole network, to indicate the existence of that group and its current status. A node that is a tree member and receives GRPH from its own upstream can use the GRPH to update their current group sequence number, current group leader and the current distance from the group leader, which requires the GRPH to be propagated from upstream to downstream step by step in its own tree structure. If a tree member receives a GRPH not from its own upstream, it first checks its group leader information recorded in its Multicast Route Table. If it is the same group leader as indicated in the GRPH, this GRPH is discarded and the node waits for next GRPH from its own upstream. If the group leader information recorded in its Multicast Route Table is not the same as indicated in the GRPH, there exists another tree with the same multicast group address but not the same group leader, and these two trees can be connected.

\subsubsection{Neighbor Connectivity Maintenance}

Tree neighbor connectivity is maintained by local repair when the downstream node of a link in the tree realizes that the link is broken by not receiving any broadcast messages from that neighbor in a specific time (usually three times of Neighbor-Hello interval). After detecting a link breakage, the downstream node first deletes that next hop (its only upstream) in its Multicast route table, and then becomes the request source node sending out RREQ-J to discover a new branch. This RREQ-J is different from the RREQ-J used for the node that is not a tree member but wants to join the multicast group, in that this RREQ-J must be broadcast and attached with an extension including additional information about the node hop count to the group leader, in order 
to avoid the old branch and its own downstream nodes responding to the RREQ-J. Besides the criteria for responding to a RREQ-J (only tree member with same or larger group sequence number can respond), when a tree member receives a RREQ-J with Extension, it must check its own hop count to the group leader, only the tree member with equal or smaller hop count can respond with a RREP-J. If the request source node tries several times (RREQ_RETRIES) to repair that branch, but has not received any RREP-J, tree partition happened due to network partition. Then, a new group leader must be selected for this partitioned tree.

\subsubsection{Group Leader Selection}

A new group leader must be selected for the partitioned tree or when the group leader revokes its group membership. When tree partition happens, and the current node is a group member, it will become the new group leader. Otherwise, it will force one of its tree neighbors to be the leader. If the current node only has one downstream node, it cancels the entry for that group in its Multicast route table, indicating it is no longer belongs to the tree, and sends out a MACT with a prune flag (MACT-P) to this downstream node, indicating that it will leave the tree and the tree needs a leader. If the current node has more than one downstream node, it selects one downstream, change its direction from downstream to upstream, and sends a MACT with a group-leader flag(MACT-GL) towards that node, indicating that it has other branch in the tree and the tree needs a leader. When receiving MACT-GL from upstream, the node changes the upstream direction into downstream. Then if the node is a group member, it will be the new group leader. Otherwise, it continues the above procedure till a group member is reached and becomes the new group leader.

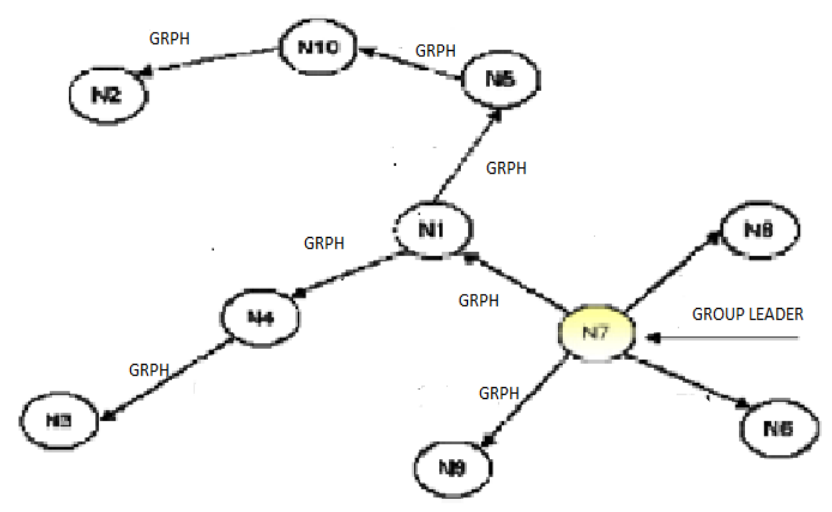

Figure 4: Group Leader in Multicast Tree

\subsubsection{Tree Merge}

Tree merge can be detected when a tree member with a smaller group leader address receives a GRPH generated by another group leader with a larger address for the same group. The tree member initiates the merge by unicastly sending a RREQ with a repair flag (RREQ-R) to the group leader, asking for the leader's permission to rebuild the tree. This RREQ-R propagates from downstream to upstream till the leader is reached. If the leader has not permitted other nodes to rebuild the tree, it can send back a RREP with a repair flag (RREP-R) to that request node. When receiving RREQ-R, the reverse route to the request node is formed, so the RREP-R follows this reverse route to the request node. One special case is that the leader itself realizes there is another tree for that group with a group leader having a larger address. In this case, the RREQ-R and RREP-R cycle is omitted and if the leader has not permitted any other tree member to rebuild 
the tree, it will start to rebuild the tree. Tree-rebuilding starts when the request node unicastly sends a RREQ with a join-and-repair flag (RREQ-JR) to the group leader with larger address.

\section{Performance Evaluation}

\subsection{Simulation}

The simulations are performed using Network Simulator 2 (NS-2), particularly popular in the wireless networking community. The performance of IH-AODV is evaluated by comparing it with MAODV protocol in same condition. In our simulation, MAC protocol is the IEEE standard 802.11 Distributed Coordination Function (DCF) [11].The traffic sources are constant bit rate (CBR). Half of nodes are static, half of nodes move with a random mobility model. For mobile nodes, velocities ranged between $0 \mathrm{~m} / \mathrm{s}$ and $20 \mathrm{~m} / \mathrm{s}$, while the pause time was set to 30 seconds. The data packet size is 512 bytes.

We conducted two sets of scenarios. First, with the number of CBR flows as 15 constantly, the nodes in the network increase from 20 to 100 gradually. The size and the area are selected .So that the nodes density is approximately constant, which would properly reflect the scalability of routing protocols. Second, we studied the performance of the protocols when the CBR flows from 15 to 40 in networks with 400 nodes. Each simulation was run for duration of 900 seconds. Each sample data we use is an average of 5 simulations. We evaluated three performance metrics:

4.1.1. Packet delivery ratio: The ratio of the data packets delivered to the destinations to those generated by the sources.

$$
\frac{\sum \text { Numberof ReceivedDataPackets }}{\sum \text { NumberofSentDataPackets }}
$$

4.1.2. Average control overhead: The control packet overhead that for route discovery, clusters maintain and route repair etc.

4.1.3. End-to-end delay: The average delay includes all possible delays caused by route discovery, propagation, and transfer times etc.

$$
\frac{\sum(\text { Timepacketarive @ dest - Time packetsent @ source })}{\text { TotalNumberofConnectionPairs }}
$$

\subsection{Results And Analysis:}

In the first scenario, we studied the scalability of the protocols. The results are shown in Fig.5. As shown in Fig. 5(a), both IH-AODV and MAODV show high packet delivery ratio even for networks with 100 nodes. But IH-AODV consistently delivers about 1-2 percent more data packets than MAODV. IH-AODV maintains routes hierarchically, an additional CMLT and repairs a broken route locally. Thus, an active route in IH-AODV usually lasts longer and more data packets can be delivered. 


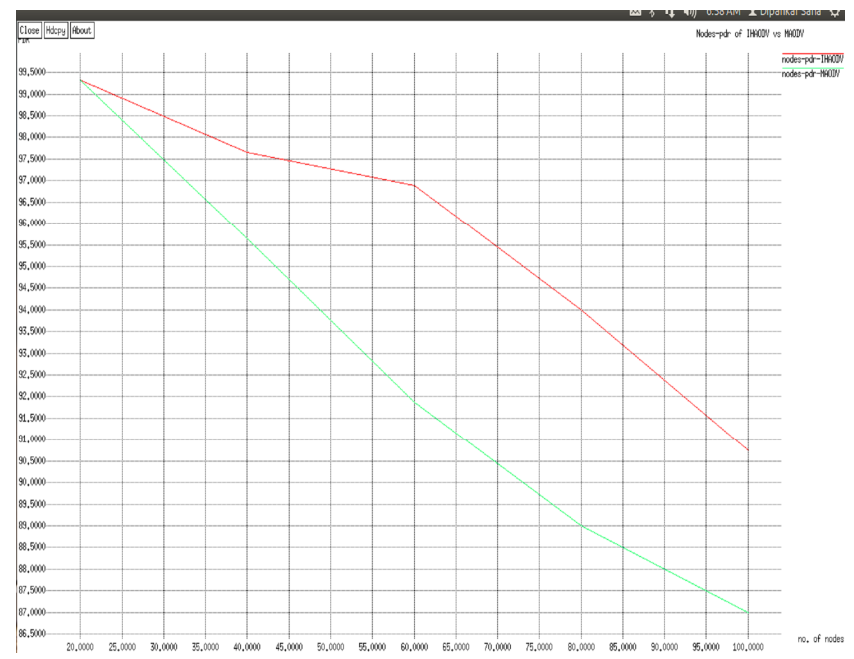

Figure 5. (a) Packet delivery ratio

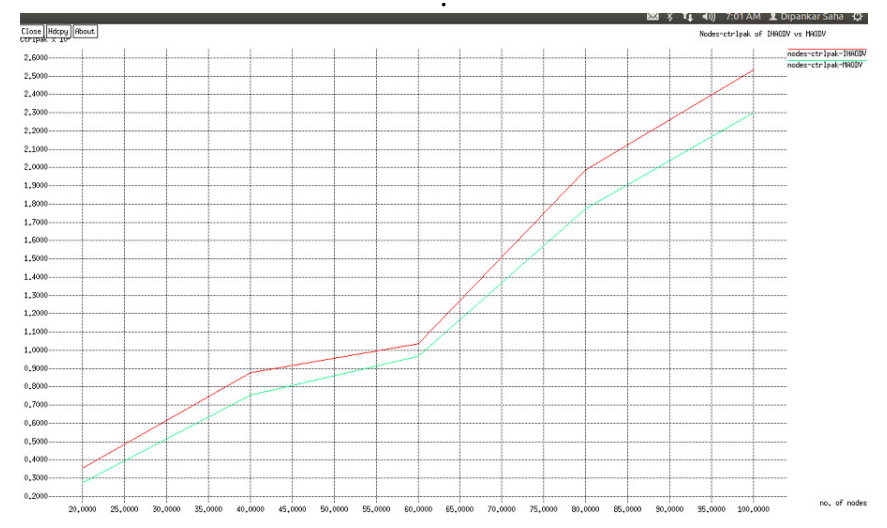

Figure 5. (b) Average control packets.

Fig. 5(b) shows the route overhead of the comparing route protocols. When nodes are above 20 then overhead of MAODV is increasing slowly with IH-AODV, when the nodes are more than 60 , the overhead of IH-AODV increase rapidly. MAODV has very light overhead as it use the multicast routing traffic. The low control overhead is critical for MAODV. 


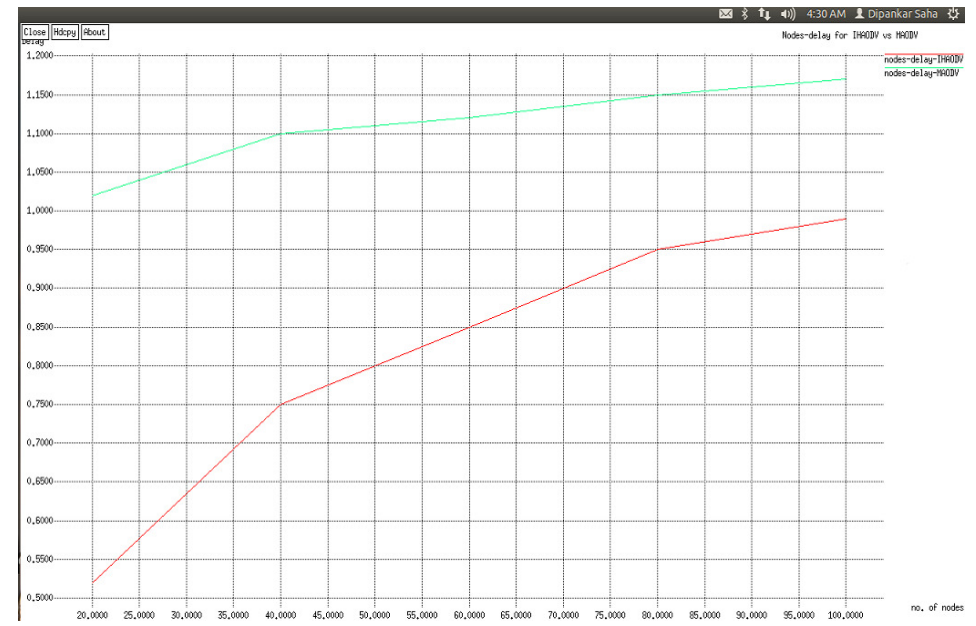

Figure.5.(c) End-to-end delay

The end-to-end delay is shown in Fig. 5(c) the network becomes large IH-AODV shows a smaller end-to-end delay in large network because the fresh route detect and route repair mechanism recovers a broken route quickly and data packets do not have to wait for another round of route discovery before they can be transmitted. The above results show that IH-AODV indeed incorporates good aspects of MAODV and hierarchical and exhibits promising performance, especially in medium to large networks. In the second scenario,

Fig. 6(a), 6(b) and 6(c) show comparison between both the route protocols on the basis of three metrics, using different number of CBR flows mentioned before.

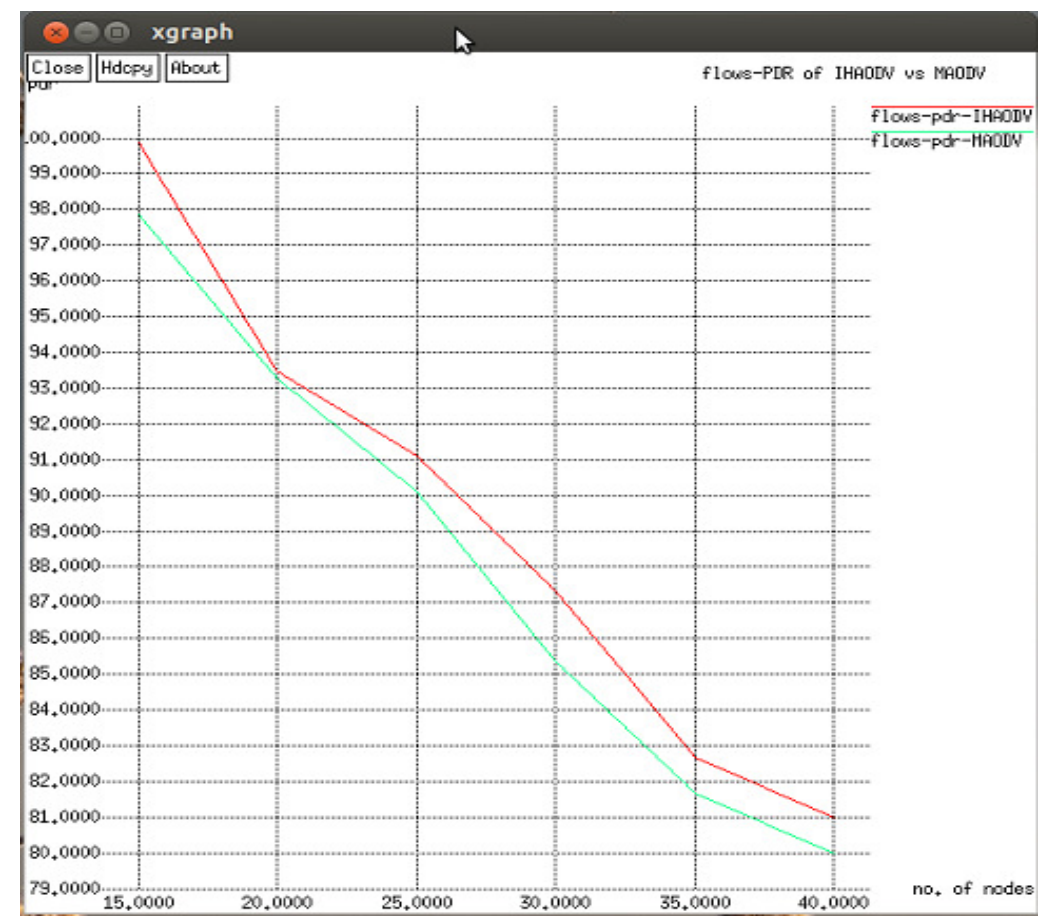

Figure 6. (a) Packet delivery ratio. 


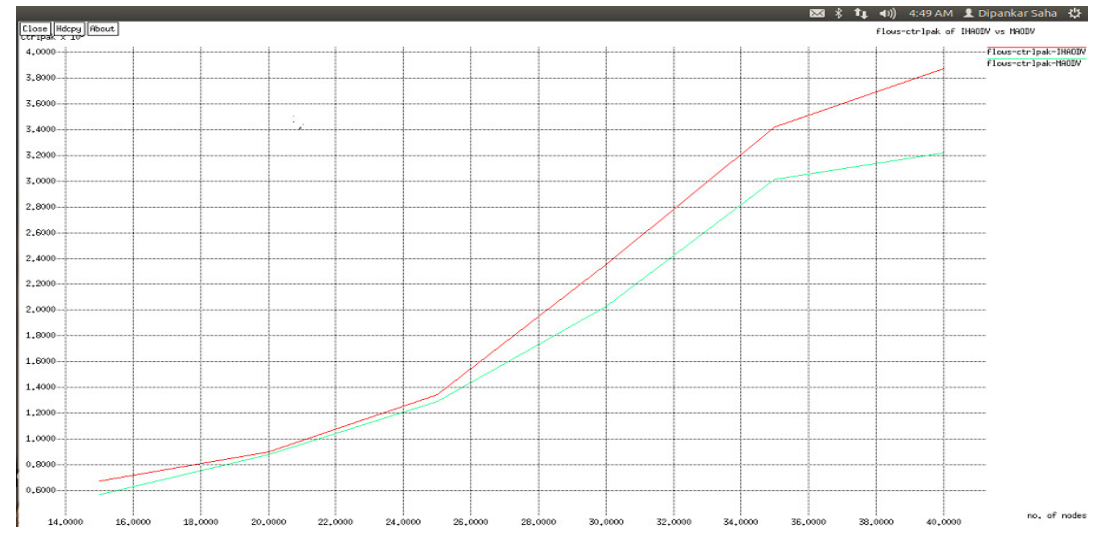

Figure 6. (b) Average control packets

Fig. 6(a) is the comparison of packet delivery ratio. In MAODV, the value drop as the number of flows increases because the network becomes more congested. Nevertheless, IH-AODV still shows well. Fig. 6(b) shows the average control overhead. We observe that MAODV can save a little control packets per flow compared to IH-AODV. This is mainly because of group hello packets propagates rapidly in the whole network.

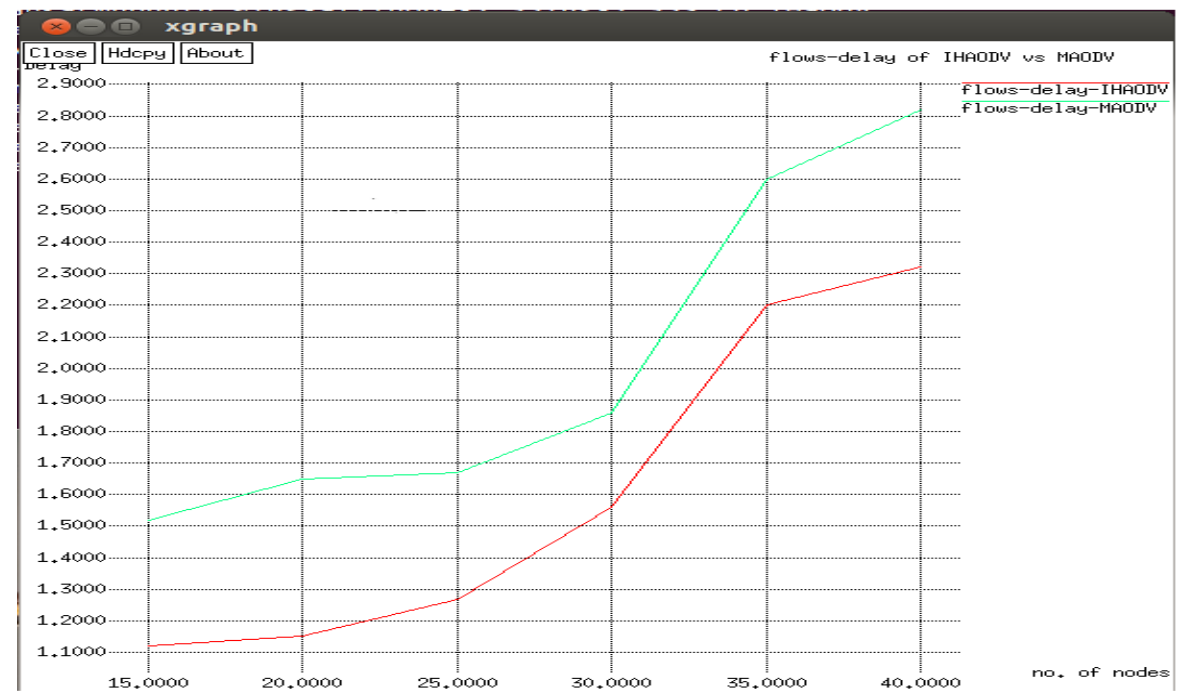

Fig: 6. (c) End-to-end delay

Fig. 4(c) shows the comparison between both the route protocols on the basis of end-to-end delay. IH-AODV is much better than MAODV while the flows increase. The reason is that the CMLT, which makes a node easier to find a route than MAODV before transmitting packets. The results show that IH-AODV works well when the network becomes congested.

\section{ConClusions}

In this paper we have implemented two routing protocols, namely reactive routing protocol Multicast Ad hoc On-Demand Distance Vector Routing (MAODV) and Improved Hierarchical routing protocol in Tool command language and integrated the module in the ns-2 [12] Simulator. We have made the performance comparison of the protocols and simulations were carried out 
with identical topologies and running different protocols on the mobile node. The performance of the protocols were measured with respect to metrics like Packet delivery ratio, end to end delay, control overhead and compared the X-graph between these two protocols. Simulation results show that IH-AODV provide better packet delivery rate with less route latency and overhead. The results confirm that our scheme achieves better scalability than MAODV with keeping the merits of it. In MAODV lower route request latency, but lower overhead than IH-AODV. IH-AODV performs better than MAODV in low mobility if the nodes increases from 20 to 60 .The size and the area are selected so that the nodes density is approximately constant, which would properly reflect the Scalability of routing protocol. Seconds we studied the performance of the protocols when the CBR flows from 15 to 40 in networks with 40 nodes. Each simulation runs for duration of 900 seconds.

\section{REFERENCES}

[1] Bruno, R. Conti, M. Gregori, E. "Mesh networks: commodity multihop ad hoc networks", IEEE Communications Magazine, Mar. 2005, Volume: 43, Issue: 3, pp: 123- 131.

[2] Ian F. Akyildiz, Xudong Wang. Weilin Wang "Wireless Mesh networks: a survey", Computer Networks Magazine, Jan. 2005, 47 (4): 445-487.

[3] J. J. Garcia-Luna-Aceves and M. Mosko, "Multipath Routing in Wireless Mesh Networks", in first IEEE Workshop on Wireless Mesh Networks (WiMesh 2005); 2005 September 26;Santa Clara; CA.

[4] C.E. Perkins and E.M. Royer, "Ad-Hoc On-Demand Distance Vector Routing", Proc. Workshop Mobile Computing Systems and Applications (WMCSA '99), Feb. 1999.

[5] Rendong Bai, Mukesh Sighal, "DOA: DSR over AODV Routing for Mobile Ad Hoc Networks", IEEE Transactions on Mobile Computing. Volume:5, No.10, Oct. 2006.

[6] D.B. Johnson and D.A. Maltz, "Dynamic Source Routing in Ad Hoc Wireless Networks", Mobile Computing, vol. 353. Kluwer Academic, 1996.

[7] Royer, E. M. and Perkins, C. E.; "Multicast Operation of the Ad-hoc On-Demand Distance Vector Routing Protocol", Proceedings of the 5th Annual ACM/IEEE International Conference on Mobile Computing and Networking (MOBICOM.99), Seattle, WA, USA, August 1999, pages 207-218 .

[8] C.-C. Chiang, H.-K. Wu, W. Liu, and M. Gerla, "Routing in Clustered Multihop Mobile Wireless Networks with Fading Channel", Proc. Singapore Int'1 Conf. Networks (SICON'97), Apr. 1997, pp. 197-211.

[9] C.E. Perkins, E.M. Belding-Royer, and I.D. Chakeres, "Ad Hoc On Demand Distance Vector (AODV) Routing", IETF Internet draft, Oct. 2003.

[10] Royer, E. M. and Perkins, C. E.; "Multicast Ad hoc On-Demand Distance Vector (MAODV) Routing", IETF, Intemet Draft: draft- ietf-manet-maodv-00.txt, 2000

[11] "Wireless LAN Medium Access Control (MAC) and Physical Layer (PHY) Specifications", IEEE Standard 802.11-1997, IEEE Standards Dept. 1994.

[12] http://www .isi.edu /nsnam/ns

\section{AUTHORS}

Debraj Modak received the bachelor's degree from WBUT of electronics and communication engineering in 2009 and master's from SRM University of Digital Communication and Networking in 2011. He has been worked as a Assistant Professor in the Institute of Science and Technology, Midnapore, during 2011-12. At present, he work as an Assistant Professor at the Department of Electronics and Communication in the Abacus Institute of Engineering and Management, Mogra.

Dipankar Saha received the bachelor's degree from WBUT of electronics and communication engineering in 2009 and master's from SRM University of Digital Communication and Networking in 2011. He worked as a lecturer in the JIS College of Engineering, Kalyani, during 2011-12. At present, he work as an Assistant Professor at the Department of Electronics and Communication in the Global Institute of Management and Technology, Krishnanagar.
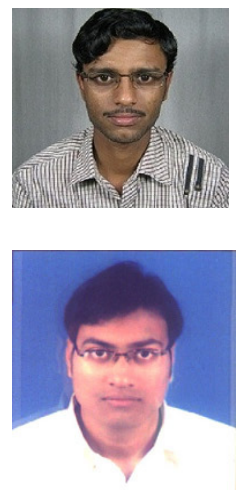